\title{
AC 2009-1211: A NOVEL PARADIGM FOR TRAINING GRADUATE STUDENTS IN SOFT SKILLS
}

\section{Sundararajan Madihally, Oklahoma State University}

Dr. Madihally is an Associate Professor in the School of Chemical Engineering at Oklahoma State University. He received his BE in ChE from Bangalore University and his $\mathrm{PhD}$ from Wayne State University in Chemical Engineering. He held a research fellow position at Massachusetts General Hospital/Harvard Medical School/Shriners Hospital for Children. His research interests include bioreactor development for tissue regeneration and the development of therapies for traumatic conditions. 


\title{
A Novel Paradigm for Training Graduate Students in Soft Skills
}

\begin{abstract}
Most chemical engineering programs which offer masters and $\mathrm{PhD}$ degrees have a common seminar series for all the graduate students. Typical seminar series includes presenters with expertise in cutting edge topics are invited. Chemical engineering is expanding and many research topics could address only a portion of the students. Further, conducting seminars for the entire duration of the semester may be cost prohibitive, leading to reduction in number of seminars. Interestingly, there are very few seminars that introduce graduate students to nontechnical content that could be paramount to their future success. For example, basics of grant writing, the importance of maintaining a laboratory notebook, writing a technical report, chemical safety demonstrations or academic integrity are not addressed. At our University, we have incorporated these topics into the seminar series in addition to presentations dealing with cultural aspects. Most of the speakers are from different departments within the University. Each seminar also included a critique (homework) submission to get the feedback on their like and dislike about the presentations. These responses have been very positive and encouraging. Students have also expressed interest in many other topics such as time management, educational research and interpersonal management. Thus, there are a number of different topics that could be useful to graduate students, which potentially minimizes redundancy for resident graduate students. In summary, the seminar series can be used as a possibility of incorporating some of the soft skills into the graduate program.
\end{abstract}

Key words: graduate program, soft skills, seminars, writing, culture

\section{INTRODUCTION.}

Graduate programs in various institutions are developed to advance the technical competency of the graduate students. As a degree requirement, graduate students enroll in few mandatory classes dealing with advanced chemical engineering topics such as thermodynamics, transport phenomena and reaction engineering. In addition, they also enroll in elective courses relevant to their research topic. PhD students are accepted as doctoral candidates upon successful completion of the qualifying exam (or preliminary exams) and research proposal defense. Students spend significant amount of time on research work with some getting an opportunity to i) present in scientific conferences and ii) publish in peer-reviewed journals. Degrees are granted after students successfully defend the research work by oral presentation and technical report.

Interestingly, role of these students significantly changes upon successful completion of their graduate degree. They are required to deal with a number of non-technical issues in addition to technical issues. For example, if they work in a research and development in the industry, they need to interact, lead or manage a group of researchers and technicians. If they become faculty members, they mentor graduate students, teach classes and write proposals despite no formal training in any of these roles. Their success in these roles is measured by their productivity and ability to bring financial resources. For example, a faculty member is measured for tenure on the number of publications in peer-reviewed journals, student evaluation from different courses they teach and success in obtaining extramural funds. These outcomes depend on the ability of a faculty member i) in coaching graduate students to be productive, ii) in teaching courses effectively, and iii) writing successful proposals. To perform these duties, they 
should have acquired soft skills such as management and writing skills. Hence, soft skills are as important as technical competencies for their success post-graduation. However, training graduate students in soft skills has been predominantly ignored with the expectation of acquiring those skills on the job or the hard way $[1,2]$.

A common course that most graduate programs have is a seminar series. Many schools have the seminar series as a mandatory course for all the graduate students. This seminar is primary used as a method of advancing their technical competencies. They are introduced to cutting edge research topics by inviting faculty members from their own institution, other institutions or industrial personnel. Typically, the presentations help reinforce technical concepts, and provide alternative research strategies or method of analyzing experimental results.

Chemical Engineering discipline has expanded to include diverse and broad range of research topics. Thus some research topics could address only a portion of graduate students in any presentation. For example, if one were to invite a bioengineering speaker whose expertise is in tissue regeneration, it may not be interesting to students whose research topic deals with thermodynamic modeling of fossil fuels. If one is dealing with nanotechnology, optimization of control systems may seem irrelevant. Thus, students may form an opinion that the seminar series is not important. In addition, inviting speakers outside the institution requires funding to cover the expenses incurred by the presenter. Thus, the number of presentations depends on the departmental funds. In some schools, there could be restricted budget and could only invite few speakers outside the institution, with the potential of not having seminars every week. In our program, we used to restrict the seminar series to six to eight invited speakers in a semester with the total of up to ten weeks of seminars instead of sixteen weeks. Thus, there were few weeks that were not utilized in every semester. To address the issue of utilizing the available time efficiently, an approach used was to introduce soft skills by inviting non-technical presenters. This study describes some of the topics discussed along with the approach adapted and the feedback from the students.

\section{DEVELOPING THE SEMINAR SCHEDULE.}

While adding new soft skill seminars, the number of invited technical presentations was kept constant from the previous semesters. To incorporate soft skills, we assessed the available resources on campus by interacting with senior faculty members in the department. With their help, those individuals were requested to present in the seminar series. To accommodate all the presentations, developing the entire schedule very early (preferably a semester before) is important. The new schedule contained five to six presentations on soft skills in addition to six to eight technical presentations. Some of the soft skills incorporated are described below:

1. Importance of technical writing. Much of the success at the graduate level hinges on academic writing skills including pre-proposal to qualify for $\mathrm{PhD}$ candidacy, dissertation, and publications in peer-reviewed journals. Upon graduation, they are expected to write successful proposals which not only include technical component but also non-technical component such as indirect cost, salaries, and benefits. To provide information regard technical writing skill, faculty members from the English department was invited for a seminar every semester. In the two seminars (one each semester) we had, some of the topics discussed include the importance of technical writing style, coherence of sentences, methodology applied while writing a technical 
paper, and usage of certain words. At the end of the seminar, students were informed about the courses available on campus which they could take if they feel a need to improve certain aspects. This has encouraged few students to enroll in those technical writing courses.

To introduce students to grant writing and the role of research administration, personnel from the college of engineering who deal with proposals were invited. In the presentation, they were introduced to topics ranging from finding available grant opportunities, various components in a grant, grant submission process through web portals such as grants.gov and management of grant post-award.

2. Safety Demonstrations. In the seminar series, one topic commonly addressed in most graduate programs is the laboratory safety. Typically, the laboratory manager or instructor responsible for undergraduate teaching laboratory performs the safety instructions. Graduate students are reminded about the importance of material safety data sheet, safe experimental practice and waste disposal constraints within the organization. However, addressing the same components every semester may not be an effective methodology, particularly for resident graduate students. Further, there are a number of safety issues one has to consider and one seminar may not be sufficient to address all the components with ever changing global issues. For example, campus safety is gaining more attention after the Virginia Tech incidence. To address these issues, one strategy we adapted was to introduce a short reminder from the laboratory manager during the first day of class. In this class, incoming new graduate students were told to meet the laboratory manager separately to learn about the procedural issues. This has helped reduce the redundant material for resident graduate students in addition to saving a day for the seminar for other safety issues. we dedicated two seminars for safety instructions.

For the two seminars (per semester), personnel from the Environmental Health and Safety (EHS) department were invited. This is coordinated by the departmental laboratory manager who regularly interacts with safety related issues. Each presentation addresses a different safety issues. For example, one seminar dealt with providing a hands-on training on fire safety (Figure 1). EHS personnel brought in the necessary equipments and organized the session in an open area. After few minutes of initial discussion, students had an opportunity to use the fire extinguisher on a gasoline fire. After-class discussions with the graduate students
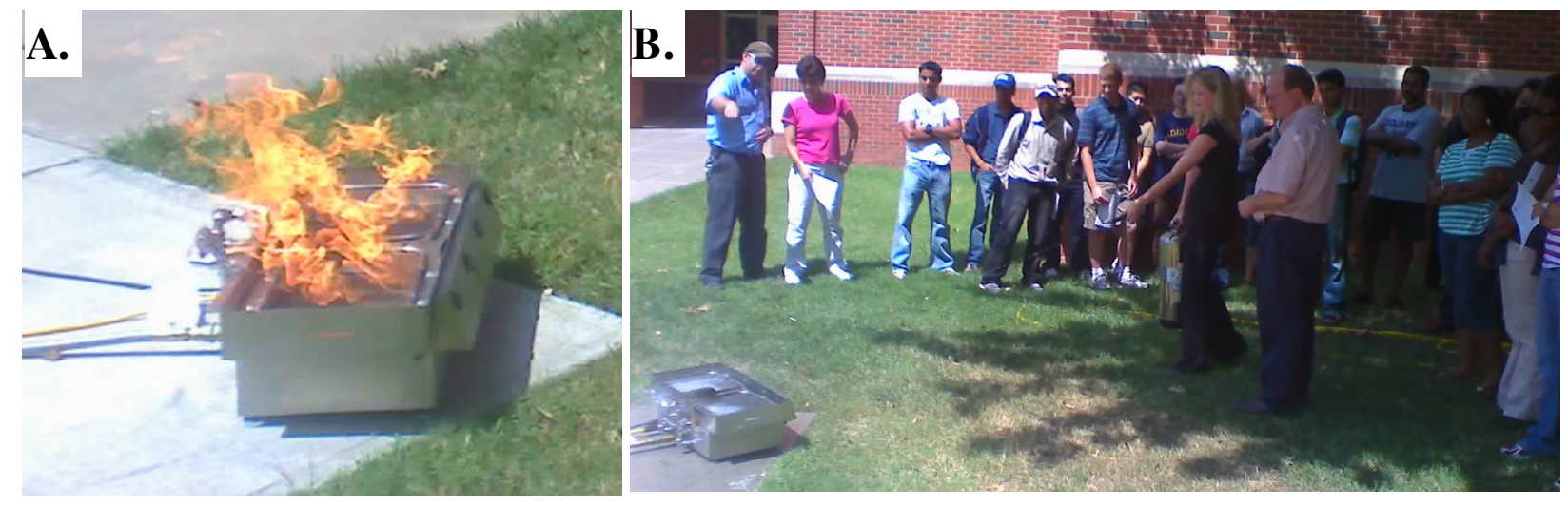

Figure 1. Fire safety demonstration. (A) Photograph of the setup used to demonstrate usage of fire extinguishers. (B) Hands-on experience of pull the pin on the fire extinguisher, aim the nozzle at the base of the fire, and then sweep from side to side (P.A.S.S) to extinguish the fire. 
suggested that they enjoyed the demonstration and would like more of those hands-on demonstrations. Other topics discussed in the seminar include work place safety and industrial safety (a video presentation entitled "Shots Fired"), good day-to-day laboratory practices, and First Aid (a video presentation). An immediate effect that the laboratory manager has observed is the significant improvement in the day to day response from the graduate students towards safety requirements. Increased awareness of safety has helped in decreasing the number of unlabeled containers and proper usage of personnel protection equipments.

3. Cultural Presentation. With increased globalization it is recognized that understanding other cultures is paramount. At the undergraduate level, study abroad programs are gaining traction in many universities with the development of departments to carry out these functions $[3,4]$. However, graduate students do not have similar opportunities although National Science Foundation has a separate directorate to promote international relations. Interestingly, most graduate program contain a wealth of diverse cultures as it is typically constituted by international students in addition to students from within United States. This provides a plethora of opportunity to understand other cultures.

To take advantage of this opportunity, a seminar has been dedicated to a cultural presentation. This seminar is schedule a week before the Finals week to provide a relaxing social environment for the graduate students. In the beginning of the semester, student volunteers from different cultures are requested. During these presentations, students are encouraged to take the help of staff members within the department and their expenses will also be reimbursed. One presentation dealt with the native American culture from a graduate students who grew-up in that environment. During the presentation, the presenter also invited tribal members and discussed various cultural aspects. There was also a demonstration of clothing and other paraphernalia utilized in various occasions. There was a discussion about the relationship between the native American involvement with the Federal government.

Another presentation was from Nigerian graduate students studying in our graduate program. Presenters came dressed in their traditional clothing (Figure 2) and prepared some snacks from the region for all the students. They presented about the history of Nigeria and their cultures. Graduate students enjoyed these interactions and there has been an overwhelming positive response to these presentations. One could think of presentations from other cultures. If the graduate program has little diversity in the population, one could get help from international
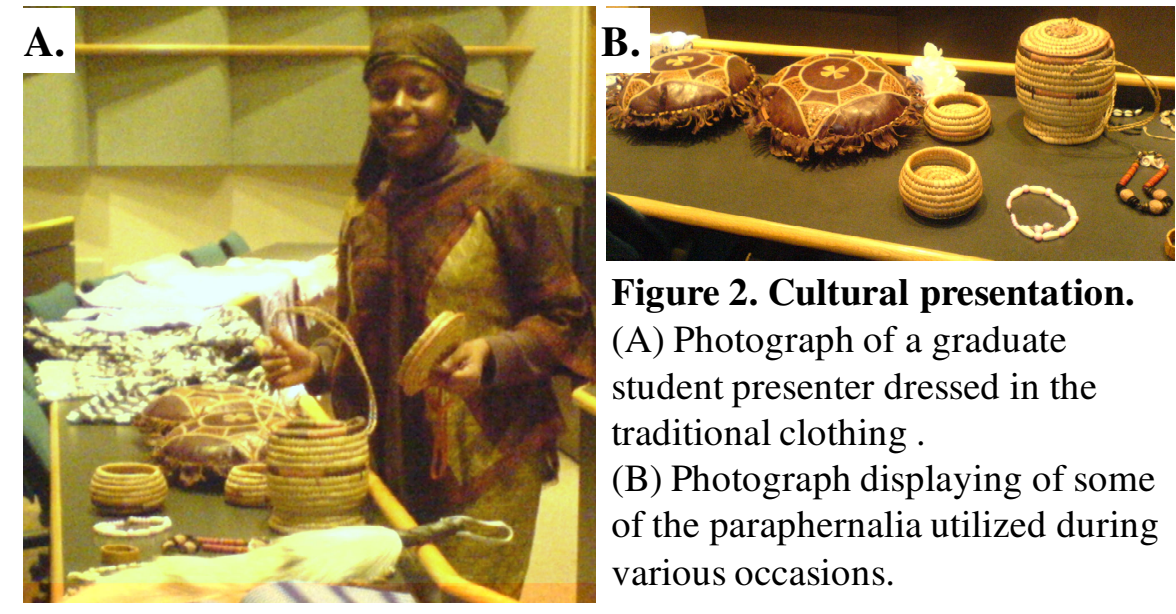

Figure 2. Cultural presentation.

(A) Photograph of a graduate student presenter dressed in the traditional clothing .

(B) Photograph displaying of some of the paraphernalia utilized during various occasions. 
services office which typically advices international student organizations. These students could be invited to give presentations.

4. Other soft skills. There are a number of other soft skills such as ethics, legal studies, management skills, economics, intellectual property protection, and teaching methodology that could be considered as seminar topics. We had few seminars on ethics, legal studies and engineering attributes. Within the department, we have a faculty member who has degrees in law as well as chemical engineering. This individual discusses topics such as importance of maintaining a laboratory notebook, issues with intellectual property, and how to apply for a patent. Another faculty member who worked in the industry prior to academic career discussed on difference between academic environment and industrial practice, and plagiarism in the modern digital world. One could rotate between topics by assessing interest of students through surveys. This also reduces the burden on the speakers who are willing to present in every semester in addition to enriching the seminar series.

\section{IMPROVING STUDENT INTEREST DURING PRESENTATION.}

Typically seminar ends with a question and answer session where the audience is allowed to ask questions to the speaker. Based on the presentation topic and the presenter, there may be few questions. Few faculty members or some graduate students working in that research area ask the question. Majority of the students do not ask questions due to multiple reasons 1) lack of interest in the topic, 2) lack of confidence in asking a question, and 3) students are not required to do anything else after the presentation i.e., there is no homework or exam on that topic.

To encourage student participation in each seminar, a novel model was adapted. They were required to submit homework for every seminar electronically through the web portal "Desired to Learn" setup for the course. This was considered towards their grade, similar to other courses. They were also given instructions about the content of the homework: a) Presentation title, b) description about what they liked in the seminar, c) description about what they did not like about the seminar, and d) other useful comments to the speaker. Students were also told that there were two alternative options to get an exemption from the homework

a) participating in the discussion (such as asking a question) at the end of that seminar and

b) presenting a seminar in the series results in exemption from five homework sets. There has been a significant increase in the number of questions at the end of technical presentations from the graduate students. There are many occasions where the instructor has cut short the session due to time constraints. Few technical presenters have given enthusiastic positive feedback on the number of questions and the quality of questions they have received. These questions also help the presenters to think of pursing other aspects in their research. However, one has to moderate the question and answer session carefully to minimize students asking redundant questions to get exemption from the homework.

When a graduate student from the program is the presenter, the comments from all the graduate students are summarized and given as a feedback to the presenter. Submitting comments electronically has helped this process to be very efficient and less time consuming. During these presentations, students who do not ask questions in external speaker presentations are encouraged to ask questions. Typically, the instructor plays an active role by identifying a specific student to ask a question. This is done to encourage public speaking. 


\section{STUDENT FEEDBACK.}

After two semesters of this approach, students were asked in a survey about their experience in the seminar series for the last two semesters. The response has been very positive. Some comments are as follows: "The seminars have become more useful and well planned. I have enjoyed most of them and learned from them. The cultural presentations are great and I like the safety seminars"; "more non technical presentations would make the seminar series more interesting"; "I have liked most of the non-technical seminars"; "asking questions should not be made a substitute for homework. I have heard a few dumb questions as a result".

When asked about how many technical to non-technical presentations they would like to have, the average response was 7.5 technical to 5.5 technical presentations in a series. This answer could be somewhat skewed as the number of technical presentations in semester is 8 and the number of non-technical presentations is 5 . Nevertheless, only one student suggested that all presentations should be technical presentations. When asked about the type of non-technical presentations they are interested in listening, following rank order was obtained based on number of votes received for each category: 1) management skills, 2) technical writing skills, 3) financial management, 4) safety skills, 5) ethics, and 6) teaching skills. Graduate students ranked the teaching skills to be not as important as management skills. This could be due to multiple factors: a) interest in pursuing an industrial job opportunity rather than an academic job, and b) lack of awareness in pedagogical research/requirement. When asked about the type of writing skills they want to learn more, majority of them indicated learning about "writing technical paper in a peer-reviewed journal" to be of interest.

\section{SUMMARY.}

Graduate seminar series provides a unique opportunity to incorporate soft skills in to the graduate program. Adapting this approach has three primary advantages:

i) making the seminar series more effective by eliminating redundancies in the schedule and utilizing the entire available time

ii) adding more value to their degree by incorporating soft skills into the graduate students, and

ii) decreasing the monitory burden on the department to invite external speakers for every seminar in the semester.

However, coordinating the seminar series requires significant time commitment from the faculty member. One has to identify the resources available on campus and coordinate the schedule. Although one could argue that a seminar in many of these topics may not be sufficient to make a graduate student proficient in soft skills, they are intended to provide an opportunity to recognize whether they have the skill set to perform these functions. In other words, the intention is similar to a technical seminar. The non-technical seminars provide an opportunity to see whether students are interested in enhancing a particular skill set. An encouraging observation is that few students have taken courses on legal studies and technical writing. Those students who have graduated and working in the industry have also given encouraging feedback on the seminar series and other topics to consider in the seminar.

\section{REFERENCES}

[1] Pulko SH and Parikh S. Teaching 'soft' skills to engineers. International Journal of Electrical Engineering Education. 40 (4): 243-254, 2003. 
[2] Kumar S, and Hsiao JK. Engineers Learn "Soft Skills the Hard Way": Planting a Seed of Leadership in Engineering Classes. Leadership and Management in Engineering. 7(1): 18-23, 2007.

[3] Harichandran R, Mason D, Prestel D, Merrill J, Streng P, Maleck T, V Galishnikova. Innovation in a Large-Scale Study Abroad Program in Engineering. Proceedings, ASEE Annual Conference, Paper 3460, 2004.

[4] Parkinson A. Engineering Study Abroad Programs: Formats, Challenges, Best Practices. Proceedings, ASEE Annual Conference, Paper 422, 2007. 